\title{
ASYMPTOTIC BEHAVIOR FOR A CLASS OF SOLUTIONS TO THE CRITICAL MODIFIED ZAKHAROV-KUZNETSOV EQUATION
}

\author{
M. PANTHEE AND M. SCIALOM
}

\begin{abstract}
We consider the initial value problem (IVP) associated to the modified Zakharov-Kuznetsov (mZK) equation

$$
u_{t}+6 u^{2} u_{x}+u_{x x x}+u_{x y y}=0, \quad(x, y) \in \mathbb{R}^{2}, t \in \mathbb{R},
$$

which is known to have global solution for given data in $u(x, y, 0)=u_{0}(x, y) \in H^{1}\left(\mathbb{R}^{2}\right)$ satisfying $\left\|u_{0}\right\|_{L^{2}}<\sqrt{3}\|\phi\|_{L^{2}}$, where $\phi$ is a solitary wave solution. In this work, the issue of the asymptotic behavior of the solutions of the modified Zakharov-Kuznetsov equation with negative energy is addressed. The principal tool to obtain the main result is the use of appropriate scaling argument from Angulo et al [4, 5].
\end{abstract}

\section{INTRODUCTION}

In this work we are interested in the initial value problem (IVP) associated to the modified Zakharov-Kuznetsov (mZK) equation

$$
\left\{\begin{array}{l}
u_{t}+6 u^{2} u_{x}+u_{x x x}+u_{x y y}=0, \quad(x, y) \in \mathbb{R}^{2}, t \in \mathbb{R} \\
u(x, y, 0)=u_{0}(x, y)
\end{array}\right.
$$

where $u=u(x, y, t)$ is a real valued function.

The well studied model that describes the weakly nonlinear waves in shallow water is the Korteweg-de Vries (KdV) equation

$$
u_{t}+u_{x x x}+u u_{x}=0, \quad x, t \in \mathbb{R} .
$$

This model also arises in several other physical contexts for example: plasma physics, stratified internal waves and ion-acoustic waves are few to mention (see [9], 8], [37] and

2000 Mathematics Subject Classification. 35Q35, 35Q53.

Key words and phrases. Korteweg-de Vries equation, Cauchy problem, Solitary wave solution, Stability.

M. P. was partially supported by FCT Portugal, through Ciência 2007 program and CMAT - Universidade do Minho, Portugal and M. S. was partially supported by FAPESP Brazil. 
references therein). Despite its apparent universality, the $\mathrm{KdV}$ equation is limited as a spatially one-dimensional model. There are several two dimensional generalizations of the $\mathrm{KdV}$ equation. The widely studied bi-dimensional generalizations of the KdV equation are the Kadomtsev-Petviashvili (KP) and the Zakharov-Kuznetsov (ZK) equations. The KP equation

$$
\left(u_{t}+u_{x x x}+u u_{x}\right)_{x}+\epsilon u_{y y}=0, \quad(x, y) \in \mathbb{R}^{2}, t \in \mathbb{R}
$$

derived by Kadomtsev and Petviashvili [17], describes the propagation of weakly nonlinear long waves on the surface of fluid, when the wave motion is essentially one-directional with weak transverse effects along y-axis. Equation (1.3) is known as KP-I for $\epsilon=-1$ and KP-II for $\epsilon=1$. On the other hand, the ZK equation

$$
u_{t}+u_{x x x}+u_{x y y}+u u_{x}=0, \quad(x, y) \in \mathbb{R}^{2}, t \in \mathbb{R},
$$

derived by Zakharov-Kuznetsov in [43], governs the behavior of weakly nonlinear ionacoustic waves in a plasma comprising cold ions and hot isothermal electrons in the presence of a uniform magnetic field (see [27, 28]). This equation, which is a more isotropic two-dimensional, was first derived for describing weakly nonlinear ion-acoustic waves in a strongly magnetized lossless plasma in two dimensions. Unlike the KP equation, the ZK equation is not integrable by the inverse scattering transform method. It was also proved in Melkonian and Maslowe [25] that the equation (1.4) to be the amplitude equation for two dimensional long waves on the free surface of a thin film flowing down a vertical plane with moderate values of the surface fluid tension and large viscosity.

The KP equation can be derived for water waves in the weakly nonlinear, weakly dispersive and weakly two dimensional limit. Although the KP equation arises in the description of physically interesting phenomena such as surface, internal, and plasma waves and has the advantage of being completely integrable using the inverse scattering transform [1], it is limited by the assumption of weak two-dimensionality.

On the other hand, the propagation of Alfvén waves at a critical angle to the undisturbed magnetic filed is described by the modified Korteweg-de Vries (mKdV) equation

$$
u_{t}+u_{x x x}+u^{2} u_{x}=0, \quad x, t \in \mathbb{R} .
$$

For detailed description we refer to Kakutani and Ono [18]. The two dimensional generalization of this equation that describes the presence of the transverse dispersion is the 
mZK equation (1.1), see Blaha and Laedke [11]. In the physical context, this phenomenon has been attributed to the finite Larmor radius effects, Hasegawa and Uberoi [16].

Our interest here is to address the asymptotic behavior of the blowing up solutions of the mZK equation (1.1) which is the critical case of the $n$-dimensional generalization of the generalized $\mathrm{KdV}$ equation

$$
u_{t}+u^{p} u_{x_{1}}+\partial_{x_{1}}\left(\nabla^{2} u\right)=0, \quad x=\left(x_{1}, x_{2}, \cdots, x_{n}\right) \in \mathbb{R}^{n}, \quad t \geq 0,
$$

where $u$ is a real function, with $p=2$ and $n=2$. Several properties of this equation including existence and stability of solitary wave solutions have extensively been studied in the literature (see for eg. [10], [13] [15], [23], [35]). Quite recently, Linares and Pastor 24] considered the Cauchy problem associated to (1.1) and proved local existence of solution for given data in $H^{s}\left(\mathbb{R}^{2}\right), s>3 / 4$, using smoothing estimates for solutions of the associated linear problem obtained in Faminskii [15] and the contraction mapping principle introduced by Kenig, Ponce and Vega [19]. Also, using the conserved quantities (see (2.3), (2.4) below) satisfied by the flow of (1.1) combined with the Gagliardo-Nirenberg interpolation inequality, they were able to obtain an a priori estimate in $H^{1}\left(\mathbb{R}^{2}\right)$ for small initial data which in turn implies global well-posedness for such data in $H^{1}\left(\mathbb{R}^{2}\right)$. This result improved the previous one by Biagioni and Linares [10]. De Bouard in [13] generalized the stability result in higher dimensions and proved that the solitary wave for (1.6) is stable if and only if $p<\frac{4}{n}$. Therefore $p=\frac{4}{n}$ is known as the critical case. In this case, when $n=2$, the equation (1.6) corresponds to the mZK equation (1.1).

In the context of plasma physics, the following equation

$$
16 u_{t}+30 u^{1 / 2} u_{x}+\nabla^{2} u_{x}=0
$$

that describes ion-acoustic waves in a cold-ion plasma when the electrons are nonisothermal, is also named as the modified ZK equation. We will not consider this model here. For details we refer to [27, 28, 34, 38] and references therein.

In 1996, Pelinovsky and Grimshaw [29] considered the instability development and critical collapse of solitary wave considered in the framework of generalized Korteweg-de Vries equation in one and two dimension. They presented a universal analytical theory for the description of the development of solitary wave instability and the related critical collapse in long-wave evolution equation. Their technique enables them to construct 
approximate solutions for self-similar critical collapse in the KdV-type evolution equation and in this case, they say, the validity of their approach is guaranteed by the result of Laedke et al in [22].

In Angulo et al [5], the work of [22] was recovered and detailed analysis of the asymptotic behavior of the blowing up solutions in the critical case $p=2 \beta+1$ for the generalized $\mathrm{KdV}$-type equation

$$
u_{t}+\left(u^{p}\right)_{x}-D^{\beta} u_{x}=0, \quad x \in \mathbb{R}, \quad t \geq 0
$$

where $\beta \geq 1$, was established.

In 2000, Sipcic and Benney [36] reviewed the results regarding the radially symmetric positive solitary wave solutions of mZK equation which are referred to as lump solutions. They studied numerically the lump interactions and illustrate how the amplitude perturbations resulting from interactions push solutions over the instability threshold. This work was a confirmation of the analytic theory of singularity formation described in Pelinovsky and Grimshaw [29].

Inspired from the results obtained in [22] and [4, 5], our work in this article addresses the asymptotic behavior of solutions of (1.1). The negative energy assumption and the spectral property in Lemma 2.4 (below) play crucial role to prove our main result. At this point, it is worth noticing that, Merle [26] proved the existence of blow-up solution in the energy space for the critical generalized KdV equation, using negative energy assumption combined with virial type identity and Liouville theorem. At least in our knowledge, there is no result of the blow-up solutions for the mZK equation, see also [24]. Generalizing results from one dimensional case to the higher dimensional case is not always trivial. If there is blow-up solution to the mZK equation (1.1) then our result shows how it occurs.

The plan of the paper is as follows. Section 2 is concerned with sharp conditions for global well-posedness of (1.1) and some preliminary results. In section 3, we state and prove the main result of this work. Section 4 is devoted to point out some concluding remarks and finally in the appendix we provide proofs of some estimates that are used in the proof of the main result.

Before leaving this section, we define some notations that will be used throughout this article. The $L^{2}$ based Sobolev space of order $s$ will be denoted by $H^{s}$ and $\|\cdot\|$ means 
$\|\cdot\|_{L^{2}\left(\mathbb{R}^{2}\right)}$. For $1 \leq p, q, r<\infty$, the mixed space-time norm is defined as

$$
\|f\|_{L_{x}^{p} L_{y}^{q} L_{T}^{r}}:=\left(\int_{\mathbb{R}}\left(\int_{\mathbb{R}}\left(\int_{0}^{T}|f(x, y, t)|^{r} d t\right)^{q / r} d y\right)^{p / q}\right)^{1 / p},
$$

with usual modifications in the case when $p=\infty, q=\infty$ or $r=\infty$.

\section{Preliminary Results}

In this section we collect some results on the initial value problem and existence of solitary wave solutions for the mZK equation. We also present some preliminary results which are needed in our further investigations. The local well-posedness result to the IVP (1.1) is stated as follows:

Theorem 2.1. For any $u_{0} \in H^{s}\left(\mathbb{R}^{2}\right), s>3 / 4$, there exist $T=T\left(\left\|u_{0}\right\|_{H^{s}}\right)>0$ and a unique solution of the IVP (1.1) associated to the $m Z K$ equation defined in the interval $[0, T]$ satisfying

$$
\begin{aligned}
& u \in C\left([0, T], H^{s}\left(\mathbb{R}^{2}\right)\right), \\
& \left\|D_{x}^{s} u_{x}\right\|_{L_{x}^{\infty} L_{y}^{2} L_{T}^{2}}+\left\|D_{y}^{s} u_{x}\right\|_{L_{x}^{\infty} L_{y}^{2} L_{T}^{2}}<\infty, \\
& \left\|\partial_{x} u\right\|_{L_{T}^{3} L_{x}^{\infty} L_{y}^{\infty}}+\left\|u_{x}\right\|_{L_{T}^{9 / 4} L_{x}^{\infty} L_{y}^{\infty}}<\infty,
\end{aligned}
$$

and

$$
\|u\|_{L_{x}^{2} L_{y}^{\infty} L_{T}^{\infty}}<\infty .
$$

The proof of this theorem can be found in Linares and Pastor [24] (see also [15] and [10] for earlier results which are sufficient for our purpose). As stated earlier, this result is proved by using smoothing estimates satisfied by the solution of the associated linear problem combined with the contraction mapping principle.

To give the statement of the global well-posedness result we will consider the unique solitary wave solution of the mZK equation defined by

$$
\phi_{c}(\xi, y)=u(x, y, t), \text { where } \xi=x-c t,
$$

which are smooth solution that vanish at infinity. Now, substituting $\phi_{c}$ in (1.1) and assuming $\phi_{c}, \phi_{c_{\xi}}, \phi_{c_{y}}, \phi_{c_{\xi \xi}}, \phi_{c_{y y}}$ tend to zero as $\xi \rightarrow \infty$ and $y \rightarrow \infty$, we have that

$$
\Delta \phi_{c}+2 \phi_{c}^{3}-c \phi_{c}=0 .
$$


Note that the equation (2.2) satisfied by the solitary wave solution 2.1 coincides with that for the stationary optical (bright) solitary waves in the two dimensional nonlinear Schrödinger equation (see [20], [30], 31]). For the existence of solution to the equation (2.2) see also [41] and [42].

Also, we record that the following quantities

$$
\begin{aligned}
& I_{1}(u(t))=\frac{1}{2} \int_{\mathbb{R}^{2}} u(t)^{2} d x d y=\frac{1}{2} \int_{\mathbb{R}^{2}} u_{0}^{2} d x d y \\
& I_{2}(u(t))=\frac{1}{2} \int_{\mathbb{R}^{2}}\left(u_{x}^{2}+u_{y}^{2}-u^{4}\right) d x d y
\end{aligned}
$$

are conserved by the flow of 1.1 .

Now we are in position to state the global existence of solutions to the IVP (1.1) that reads:

Theorem 2.2. Let $u_{0} \in H^{1}\left(\mathbb{R}^{2}\right)$. If $\left\|u_{0}\right\|_{L^{2}}<\sqrt{3}\|\phi\|_{L^{2}}, \phi=\phi_{c}$, for some $c$ as in 2.1), then the solution $u$ of the IVP (1.1) given by Theorem 2.1 can be extended to any interval of time $[0, T]$.

The proof of this theorem follows using an $H^{1}\left(\mathbb{R}^{2}\right)$-a priori estimate that can be obtained from conservations laws $(2.3)$ and $(2.4)$ and the Gagliardo-Nirenberg interpolation inequality, see Linares and Pastor [24].

We observe that if $I_{1}^{\prime}$ and $I_{2}^{\prime}$ represent the Frechet derivatives of $I_{1}$ and $I_{2}$, then we have

$$
\begin{aligned}
\left\langle I_{1}^{\prime}\left(\phi_{c}\right), v\right\rangle & =\lim _{h \rightarrow 0} \frac{I_{1}\left(\phi_{c}+h v\right)-I_{1}\left(\phi_{c}\right)}{h} \\
& =\left\langle\phi_{c}, v\right\rangle . \\
\left\langle I_{2}^{\prime}\left(\phi_{c}\right), v\right\rangle & =\lim _{h \rightarrow 0} \frac{I_{2}\left(\phi_{c}+h v\right)-I_{2}\left(\phi_{c}\right)}{h} \\
& =\left\langle-\Delta \phi_{c}-2 \phi_{c}^{3}, v\right\rangle .
\end{aligned}
$$

Therefore, in view of 2.2 we get,

$$
I_{2}^{\prime}\left(\phi_{c}\right)+c I_{1}^{\prime}\left(\phi_{c}\right)=-\Delta \phi_{c}-2 \phi_{c}^{3}+c \phi_{c}=0
$$


Let $H_{c}$ be the linearized operator of $I_{2}^{\prime}+c I_{1}^{\prime}$ around $\phi_{c}$, namely $H_{c}=I_{2}^{\prime \prime}\left(\phi_{c}\right)+c I_{1}^{\prime \prime}\left(\phi_{c}\right)$. Now,

$$
\begin{aligned}
\left\langle I_{1}^{\prime \prime}\left(\phi_{c}\right)(v), w\right\rangle & =\lim _{h \rightarrow 0} \frac{\left\langle I_{1}^{\prime}\left(\phi_{c}+h v\right), w\right\rangle-\left\langle I_{1}^{\prime}\left(\phi_{c}\right), w\right\rangle}{h} \\
& =\lim _{h \rightarrow 0} \frac{\left\langle\phi_{c}+h v, w\right\rangle-\left\langle\phi_{c}, w\right\rangle}{h} \\
& =\langle v, w\rangle . \\
\left\langle I_{2}^{\prime \prime}\left(\phi_{c}\right)(v), w\right\rangle= & \lim _{h \rightarrow 0} \frac{\left\langle I_{2}^{\prime}\left(\phi_{c}+h v\right), w\right\rangle-\left\langle I_{2}^{\prime}\left(\phi_{c}\right), w\right\rangle}{h} \\
= & \lim _{h \rightarrow 0} \frac{\left\langle-\Delta\left(\phi_{c}+h v\right)-\left(\phi_{c}+h v\right)^{3}, w\right\rangle-\left\langle-\Delta \phi_{c}-2 \phi_{c}^{3}, w\right\rangle}{h} \\
= & \left\langle\left(-\Delta-6 \phi_{c}^{2}\right)(v), w\right\rangle .
\end{aligned}
$$

Hence, we obtain,

$$
\left\langle H_{c} v, w\right\rangle=\left\langle\left(I_{2}^{\prime \prime}\left(\phi_{c}\right)+c I_{1}^{\prime \prime}\left(\phi_{c}\right)\right)(v), w\right\rangle=\left\langle\left(-\Delta-6 \phi_{c}^{2}+c\right) v, w\right\rangle .
$$

Therefore, in particular, the equation (2.6) along with (2.2) yield

$$
H_{c}\left(\partial_{\xi} \phi_{c}\right)=0 .
$$

To proceed further with our analysis we need some hypotheses on $\phi_{c}$ and $H_{c}$.

Hypothesis 1 -(existence of solitary wave). There is an interval $\left(c_{1}, c_{2}\right) \subset \mathbb{R}$ such that for any $c \in\left(c_{1}, c_{2}\right)$, there exists a solution $\phi_{c}$ of $(2.2)$ in $H^{1}\left(\mathbb{R}^{2}\right)$. The curve $c \mapsto \phi_{c}$ for $c>0$ is $C^{1}$ with values in $H^{2}\left(\mathbb{R}^{2}\right)$. Moreover $(1+|\xi|)^{1 / 2} \frac{d}{d c} \phi_{c}, \Delta \phi_{c} \in L^{1}\left(\mathbb{R}^{2}\right)$.

Hypothesis 2 - The null space of the operator $H_{c}$ is spanned by the functions $\partial_{\xi} \phi_{c}$ and $\partial_{y} \phi_{c}$, i.e., the only zero modes of $H_{c}$ are $\partial_{\xi} \phi_{c}$ and $\partial_{y} \phi_{c}$. $H_{c}$ has a unique negative simple eigenvalue with eigenfunction $\psi_{c}$. Besides the negative and zero eigenvalues, the rest of the spectrum of $H_{c}$ is positive and bounded away from zero. Moreover the curve $c \mapsto \psi_{c}$ is continuous with values in $H^{2}\left(\mathbb{R}^{2}\right),\left\langle\phi_{c}, \psi_{c}\right\rangle \neq 0$ and $(1+|\xi|)^{1 / 2} \psi_{c}, \Delta \psi_{c} \in L^{1}\left(\mathbb{R}^{2}\right)$.

Lemma 2.3. Hypotheses 1 and 2 are true for the case of the modified Zakharov-Kuznetsov equation (1.1). 
Proof. Since the equation (2.2) coincides with that for the solitary waves for the two dimensional nonlinear Schrödinger (NLS) equation, the hypotheses on the existence follows from that for the NLS equation, see Weinstein [41, 42]. The spectral properties follow from the positivity property of the operator $-\Delta+c$ and the assumption on $\phi_{c}$, see for example, [2], 3], [13], [41] or [42].

Under the spectral hypothesis of the operator $H_{c}$ stated above we have the following result which is crucial to prove our main result of this work.

Lemma 2.4. Let $h=\phi-\sigma \Delta \phi ; \sigma<0, \phi=\phi_{c}$ for some $c$, then

$$
\inf \left\{\left\langle H_{c} f, f\right\rangle:\|f\|_{L^{2}}=1, \quad\langle h, f\rangle=0, \quad f \perp \phi^{2} \phi_{x_{i}}, i=1,2\right\}=\nu>0 .
$$

Proof. The proof of this lemma follows the same lines of arguments used to obtain similar results in Angulo et al in [4, 5], so we omit the details.

\section{Stability theorem for the MZK in the CRitical CASE}

To study the asymptotic behavior of the blowing up solutions of the mZK equation in the critical case, we follow the idea introduced in Laedke et al [22] and Angulo et al [4, 5]. Let us start by introducing the following auxiliary function

$$
\psi(x, y, t)=\mu(t)^{-1} u\left(\mu(t)^{-1} x, \mu(t)^{-1} y, t\right)
$$

where $\mu(t)$ is given by

$$
\mu(t)=\frac{\|\nabla u(t)\|}{\left\|\nabla \phi_{c}\right\|}
$$

and $0 \leq t \leq t^{*}$, with $t^{*}$ the maximal time of existence of the solution $u$ of $(1.1)$. It is immediate to verify that the function $\psi(x, y, t)$ satisfies the identities

$$
\left.\begin{array}{l}
\text { i) } \quad I_{1}(\psi(\cdot, \cdot, t))=I_{1}\left(u_{0}\right) \\
\text { ii) } \quad I_{2}(\psi(\cdot, \cdot, t))=\frac{1}{\mu^{2}(t)} I_{2}\left(u_{0}\right) \\
\text { iii) }\langle\psi(\cdot, \cdot, t), \Delta \psi(\cdot, \cdot, t)\rangle=\left\langle\phi_{c}, \Delta \phi_{c}\right\rangle
\end{array}\right\} .
$$

The following lemma provides the regularity property of the auxiliary function $\psi$ whose proof follows from the facts that $\mu \in C\left(\left[0, t^{*}\right) ; \mathbb{R}\right)$ and $0<\mu(t)<\infty$ for $0 \leq t<t^{*}$. 
Lemma 3.1. If $u \in C\left(\left[0 ; t^{*}\right), H^{1}\left(\mathbb{R}^{2}\right)\right)$, then

$$
\psi \in C\left(\left[0, t^{*}\right), H^{1}\left(\mathbb{R}^{2}\right)\right)
$$

The notion of stability we are considering in this work is with respect to the form, i.e., up to translation in space. Therefore, to deal with it we introduce the orbit

$$
\mathcal{O}\left(\phi_{c}\right)=\left\{g \mid g=\tau_{\left(r_{1}, r_{2}\right)} \phi_{c} \text { for some }\left(r_{1}, r_{2}\right) \in \mathbb{R}^{2}\right\},
$$

where $\tau_{\left(r_{1}, r_{2}\right)}$ is the translation operator defined by

$$
\left(\tau_{\left(r_{1}, r_{2}\right)} f\right)(x, y)=f\left(x+r_{1}, y+r_{2}\right), \text { for all }(x, y) \in \mathbb{R}^{2}
$$

Now, to measure the deviation of $\psi$ from the orbit $\mathcal{O}\left(\phi_{c}\right)$ in $H^{1}\left(\mathbb{R}^{2}\right)$, we introduce the pseudo-metric $\rho_{c}$ defined by

$$
\begin{aligned}
\rho_{c}\left(\psi(\cdot, \cdot, t), \phi_{c}\right)^{2}=\inf _{\left(r_{1}, r_{2}\right) \in \mathbb{R}^{2}}\{ & \left\|\tau_{\left(r_{1}, r_{2}\right)} \nabla \psi(\cdot, \cdot, t)-\nabla \phi_{c}(\cdot, \cdot)\right\|^{2} \\
& \left.+c\left\|\tau_{\left(r_{1}, r_{2}\right)} \psi(\cdot, \cdot, t)-\phi_{c}(\cdot, \cdot)\right\|^{2}\right\} .
\end{aligned}
$$

Finally, let us define the set $\mathcal{K}$ as

$$
\mathcal{K}=\left\{u_{0} \in H^{1}\left(\mathbb{R}^{2}\right) \text { such that } I_{2}\left(u_{0}\right) \leq 0\right\}
$$

Observe that if $\left\|u_{0}\right\| \leq\left\|\phi_{c}\right\|$ for some $c$ then we have $I_{2}\left(u_{0}\right) \geq 0$. This observation follows by using the sharp interpolation estimate established in Weinstein [39].

Now, we are in the position to state the main result of this work that deals with the spatial structure of the solution to the mZK equation (1.1).

Theorem 3.2. Let $\phi=\phi_{c}, c>0$ be a solitary wave solution of (1.1). For any $\epsilon>0$, there is a $\delta(\epsilon)>0$ such that if $u_{0} \in \mathcal{K}$ with $\rho_{c}\left(u_{0}, \phi_{c}\right)<\delta$ and $u$ is the solution of (1.1) with initial data $u_{0}$, then $u \in C\left(\left[0, t^{*}\right), H^{1}\left(\mathbb{R}^{2}\right)\right)$ and

$$
\begin{aligned}
\inf _{\left(r_{1}, r_{2}\right) \in \mathbb{R}^{2}}\{c & \left\|u(\cdot, \cdot, t)-\mu(t) \tau_{\left(r_{1}, r_{2}\right)} \phi(\mu(t)(\cdot, \cdot))\right\|^{2} \\
& \left.+\frac{1}{\mu^{2}(t)}\left\|\nabla u(\cdot, \cdot, t)-\mu^{2}(t) \tau_{\left(-r_{1},-r_{2}\right)} \nabla \phi(\mu(t)(\cdot, \cdot))\right\|^{2}\right\}<\epsilon
\end{aligned}
$$

for all $t \in\left[0, t^{*}\right)$, where $t^{*}$ is the maximal existence time for the solution $u$ and $\mu$ is as given in 3.2 . 
Proof: Initially we assume $\mu(0)=1$, the general case will be treated later on. The proof is based on the time dependent functional

$$
B_{t}[u]=\frac{1}{\mu^{2}(t)} I_{2}(u(t))+\frac{c}{2}\left(\frac{\|u(t)\|}{\|\phi\|}\right)^{2 k}\left(\|u\|^{2}-\|\phi\|^{2}\right)
$$

where the natural number $k$ will be chosen later. Note that, in view of the conserved energy and charge, $B_{t}[u]=B_{t}\left[u_{0}\right]$. Also, in terms of $\psi$, we have that

$$
\widetilde{B}_{t}[\psi]:=I_{2}(\psi)+\frac{c}{2}\left(\frac{\|\psi\|}{\|\phi\|}\right)^{2 k}\left(\|\psi\|^{2}-\|\phi\|^{2}\right)
$$

where the explicit dependence on $\mu$ disappears.

Using the argument described in [4, 5], the proof of the Theorem 3.2 follows, if we can prove, modulo spatial translation, the inequalities

$$
\Delta \widetilde{B}_{t} \leq c_{0}\left\|u_{0}-\phi\right\|,
$$

and

$$
\begin{aligned}
\Delta \widetilde{B}_{t} \geq c_{1}\|\psi(\cdot, \cdot, t)-\phi(\cdot, \cdot)\|_{H^{1}\left(\mathbb{R}^{2}\right)}^{2}-c_{2} \sum_{j=1}^{2}\|\psi(\cdot, \cdot, t)-\phi(\cdot, \cdot)\|_{H^{1}\left(\mathbb{R}^{2}\right)}^{j+2} \\
\quad-\sum_{j=1}^{2 k} c_{k j}\|\psi(\cdot, \cdot, t)-\phi(\cdot, \cdot)\|_{H^{1}\left(\mathbb{R}^{2}\right)}^{j+2},
\end{aligned}
$$

where $\Delta \widetilde{B}_{t}=\widetilde{B}_{t}[\psi]-\widetilde{B}_{t}[\phi]$, and $c_{i}, c_{k j}$ are fixed constants.

The proofs of the estimates (3.7) and (3.8) will be presented in the Appendix. Now we show that the estimates (3.7) and (3.8) imply the proof of the theorem. For this, let $u_{0} \in \mathcal{K}$ with $\left\|u_{0}-\phi_{c}\right\|_{H^{1}}=\delta$ and $\tilde{T}$ suitably chosen, for example as in A.17 in the Appendix. Then the estimates (3.7) and (3.8) yield, for $t \in[0, \tilde{T}]$ that,

$$
q\left(\rho_{c}\left(\psi(\cdot, \cdot, t), \phi_{c}(\cdot, \cdot)\right) \leq \Delta \widetilde{B}_{t} \leq c_{0} \delta\right.
$$

where $q(x)=\widetilde{c}_{1} x^{2}-\widetilde{c}_{2} \sum_{j=1}^{2} \ell_{j}(c) x^{j+2}-\sum_{j=1}^{2 k} d_{k, j} x^{j+2}$.

Since $\|a(\cdot, \cdot, t)\|_{H^{1}}^{2}=\rho_{c}\left(\psi(\cdot, \cdot, t), \phi_{c}(\cdot, \cdot)\right)^{2}$ is continuous function of $t \in\left[0, t^{*}\right)$, it follows from the inequality

$$
q\left(\rho_{c}\left(\psi(\cdot, \cdot, 0), \phi_{c}(\cdot, \cdot)\right) \leq c_{0} \delta\right.
$$


and 3.9 , that given $\epsilon>0$, for all $t \in[0, \widetilde{T}]$,

$$
\rho_{c}\left(\psi(\cdot, \cdot, t), \phi_{c}(\cdot, \cdot)\right) \leq \epsilon
$$

provided that $\delta$ is chosen small enough. It can be shown that the estimate 3.10 holds for all $t \in\left[0, t^{*}\right)$ (see Appendix) and this completes the proof in the case $\mu(0)=1$. The general case where the initial data is such that $\mu(0) \neq 1$ can be treated exactly as in Angulo et al [5], so we omit the details.

\section{Concluding Remarks}

Asymptotic behavior of wave propagation i.e., the phenomenon of wave collapse or blow-up in finite time for many generalized models has been a subject of study in the recent years. There are several works to address this subject in the critical cases of the nonlinear Schrödinger equation and the KdV equation in the literature, see for example [4], [5], [11], [22], 29], [36] and references therein. Our main result in this work proves that the solutions to the mZK equation $(1.1)$ which are initially close to a lump solitary wave, and which may blow-up, do so according the scaling law. In other words, if there exists a blow-up solution for the mZK equation (1.1) then the blow-up occurs along the solitary wave. Our result is in accordance with the one predicted numerically in Pelinovsky and Grimshaw 29] and Sipcic and Benney [36]. The scaling parameter $\mu$ is an auxiliary tool that depends on the solution $u$. Note that, if one can prove the existence of blow-up solution in finite time for the mZK equation (1.1) then the scaling parameter $\mu(t)$ may also blow-up in finite time, which is the case while dealing with the NLS and the KdV equations in the critical cases, see Laedke et al [22]. Finally, we would like to point that there is an extensive numerical study about the self-similar blow-up solutions of the generalized KdV equation in Dix and McKinney [14] that may serve as a motivation to conduct a similar study for the model considered here.

\section{Appendix A. Estimates used in the proof of the main RESUlt}

In this section we will prove the estimates (3.7), (3.8) and the validity of the inequality (3.10) for all $t \in\left[0, t^{*}\right)$.

Lemma A.1. The estimate (3.7) holds true. 
Proof. The proof follows using the fact that $u_{0} \in \mathcal{K}$, i.e., $I_{2}\left(u_{0}\right) \leq 0$ and $I_{2}(\phi) \geq 0$. Note that the constant $c_{0}$ in $(3.7)$ depends on $\|\phi\|$.

Lemma A.2. The estimate (3.8) holds true.

Proof. To establish this estimate we start considering the perturbation of the solitary wave solution $\phi=\phi_{c}$

$$
\psi\left(x+\gamma_{1}, y+\gamma_{2}, t\right)=\phi(x, y)+a(x, y, t)
$$

where $\left(\gamma_{1}, \gamma_{2}\right)=\gamma(t)$ will be chosen later so as to minimize the functional

$$
\Pi_{t}\left(\gamma_{1}, \gamma_{2}\right)=\left\|\nabla \psi\left(\cdot+\gamma_{1}, \cdot+\gamma_{2}, t\right)-\nabla \phi(\cdot, \cdot)\right\|^{2}+c\left\|\psi\left(\cdot+\gamma_{1}, \cdot+\gamma_{2}, t\right)-\phi(\cdot, \cdot)\right\|^{2} .
$$

Using A.11, the definition of $H_{c}$, Cauchy Schwartz inequality and Gagliardo-Nirenberg interpolation estimate we can obtain

$$
\begin{aligned}
\Delta \widetilde{B}_{t} & =\widetilde{B}_{t}[\phi+a]-\widetilde{B}_{t}[\phi] \\
& =I_{2}(\phi+a)-I_{2}(\phi)+\frac{c}{2}\left(\frac{\|\phi+a\|}{\|\phi\|}\right)^{2 k}\left(\|\phi+a\|^{2}-\|\phi\|^{2}\right) \\
& \geq\left\langle H_{c} a, a\right\rangle+\frac{4 c k}{\|\phi\|^{2}}\langle\phi, a\rangle^{2}-\sum_{j=1}^{2} d_{j}(c)\|a\|_{H^{1}}^{j+2}-\sum_{j=3}^{2 k+2} d_{k j}(c)\|a\|_{H^{1}}^{j},
\end{aligned}
$$

where $d_{j}(c)$ and $d_{k j}(c)$ are some positive constants.

The next lemma deals with obtaining a suitable lower bound on the functional $\Pi_{t}$ given in $\mathrm{A} .12$.

Lemma A.3. Suppose that for some $t \in\left[0, t^{*}\right)$ and some $\widetilde{\gamma}=\left(\widetilde{\gamma}_{1}, \widetilde{\gamma}_{2}\right) \in \mathbb{R} \times \mathbb{R}$ it is the case that

$$
\Pi_{t}(\widetilde{\gamma})<\left\|(\Delta+c)^{1 / 2} \phi_{c}\right\|^{2}
$$

Then it follows that

$$
\inf \left\{\Pi_{t}\left(\gamma_{1}, \gamma_{2}\right):\left(\gamma_{1}, \gamma_{2}\right) \in \mathbb{R} \times \mathbb{R}\right\}
$$

is attained at least once in $\mathbb{R} \times \mathbb{R}$.

Proof. Let $\gamma=\left(\gamma_{1}, \gamma_{2}\right)$. It is clear that $\Pi_{t}(\gamma)$ is a continuous function of $\gamma \in \mathbb{R}^{2}$. Moreover

$$
\begin{aligned}
\lim _{|\gamma| \rightarrow \infty} \Pi_{t}(\gamma) & =\|\nabla \psi(t)\|^{2}+\|\nabla \phi\|^{2}+c\|\psi(t)\|^{2}+c\|\phi\|^{2} \\
& =\left\|(\Delta+c)^{1 / 2} \phi\right\|^{2}+\|\nabla \phi\|^{2}+c\left\|u_{0}\right\|^{2}
\end{aligned}
$$


Therefore, hypothesis A.14, the continuity of $\Pi_{t}(\gamma)$ and A.16 lead to desired result.

Next we need to obtain the hypothesis (A.14 is satisfied for all $t$ in some interval. Let $\epsilon>0$ be such that

$$
\epsilon^{2}<\frac{1}{2} \max \{1, c\}\left\|(\Delta+c)^{1 / 2} \phi\right\|^{2} .
$$

The solitary wave is globally defined and hence by the continuous dependence theory (see [10]) for $\epsilon$ and $T>0$, there exists a $\delta>0$ such that if $\left\|u_{0}-\phi\right\|_{H^{1}}<\delta$ then the corresponding solution of 1.1 exists at least for $0 \leq t \leq T$ and

$$
\|u(\cdot, \cdot, t)-\phi(\cdot-c t, \cdot)\|_{H^{1}\left(\mathbb{R}^{2}\right)}<\epsilon / 2
$$

for all $0 \leq t \leq T$. From the continuity of $\psi$ and $u$ it follows that there is a $T_{1}>0$ such that for all $t \in\left[0, T_{1}\right]$,

$$
\|\psi(\cdot, \cdot, t)-\psi(\cdot, \cdot, 0)\|_{H^{1}\left(\mathbb{R}^{2}\right)} \leq \epsilon / 4
$$

and

$$
\|u(\cdot, \cdot, t)-u(\cdot, \cdot, 0)\|_{H^{1}\left(\mathbb{R}^{2}\right)} \leq \epsilon / 4 .
$$

Then for $0 \leq t \leq \min \left\{T, T_{1}\right\}=\tilde{T}$ (say),

$$
\begin{aligned}
\|\psi(\cdot, \cdot, t)-\phi(\cdot-c t, \cdot)\| & \leq\|\psi(\cdot, \cdot, t)-u(\cdot, \cdot, t)\|+\|u(\cdot, \cdot, t)-\phi(\cdot-c t, \cdot,)\| \quad \text { A.17) } \\
& \leq\|\psi(\cdot, \cdot, t)-\psi(\cdot, \cdot, 0)\|+\|u(\cdot, \cdot, 0)-u(\cdot, \cdot, t)\|+\epsilon / 2=\epsilon .
\end{aligned}
$$

Thus, the infimum in A.15 is taken on at finite values $\gamma(t)$ throughout some time interval viz. $[0, \tilde{T}]$.

Now, a compatibility relation is obtained by differentiating $\Pi_{t}(\gamma)$ with respect to $\gamma_{1}$ and $\gamma_{2}$ and evaluating at values that minimize $\Pi_{t}(\gamma)$

$$
\begin{aligned}
& \frac{d}{d \gamma_{1}}\left[\int_{\mathbb{R}^{2}}\left(\left|\psi_{x}\left(x+\gamma_{1}, y+\gamma_{2}, t\right)-\phi_{x}(x, y)\right|^{2}+\left|\psi_{y}\left(x+\gamma_{1}, y+\gamma_{2}, t\right)-\phi_{y}(x, y)\right|^{2}\right) d x d y\right. \\
& \left.\quad+c \int_{\mathbb{R}^{2}}\left(\left|\psi\left(x+\gamma_{1}, y+\gamma_{2}, t\right)-\phi(x, y)\right|^{2}\right) d x d y\right] \\
& =2 \int_{\mathbb{R}^{2}}\left[a_{x} \phi_{x x}+a_{y} \phi_{x y}+c a \phi_{x}\right] d x d y .
\end{aligned}
$$

Using $-\Delta \phi-2 \phi^{3}+c \phi=0$, we obtain the first compatibility condition

$$
\int_{\mathbb{R}^{2}} a \phi^{2} \phi_{x} d x d y=0
$$


Analogously we can obtain the second compatibility condition

$$
\int_{\mathbb{R}^{2}} a \phi^{2} \phi_{y} d x d y=0
$$

The issue to obtain a lower bound $(3.8)$ for the right-hand side of inequality $(\mathrm{A} .13)$ is the next goal. Attention is now turned to estimating the term

$$
\left\langle H_{c} a, a\right\rangle+\frac{4 k c}{\|\phi\|^{2}}(\langle a, \phi\rangle)^{2}
$$

where $a$ satisfies the compatibility relations A.18 and A.19.

Let $a_{\|}=\frac{\langle a, h\rangle}{\|h\|^{2}} h$ and $a_{\perp}=a-a_{\|}$, where $h=\phi-\sigma \Delta \phi$ with $\sigma<0$ as in Lemma 2.4. It follows from properties of $a$ and $h$ that $\left\langle a_{\perp}, h\right\rangle=0$. Now, together with this orthogonality relation and compatibility conditions in (A.18) and A.19) we can use Lemma 2.4 to obtain

$$
\begin{aligned}
\left\langle H_{c} a_{\perp}, a_{\perp}\right\rangle \geq D_{1}\left\|a_{\perp}\right\|^{2}, \quad D_{1}>0 . & \\
\left\langle H_{c} a_{\|}, a_{\|}\right\rangle & =\left\langle H_{c} \frac{\langle a, h\rangle h}{\|h\|^{2}}, \frac{\langle a, h\rangle h}{\|h\|^{2}}\right\rangle \\
& =\frac{\langle a, h\rangle^{2}}{\|h\|^{4}}\left\langle H_{c} h, h\right\rangle=\frac{\left\|a_{\|}\right\|^{2}}{\|h\|^{2}}\left\langle H_{c} h, h\right\rangle \\
2\left\langle H_{c} a_{\|}, a_{\perp}\right\rangle & =2 \frac{\langle a, h\rangle}{\|h\|^{2}}\left\langle H_{c} h, a_{\perp}\right\rangle \geq-D_{2}\left\|a_{\perp}\right\|\left\|a_{\|}\right\|
\end{aligned}
$$

for some positive constants $D_{1}$ and $D_{2}$. Thus, from the Cauchy-Schwartz inequality we can get

$$
\begin{aligned}
\frac{4 k c}{\|\phi\|^{2}}\langle a, \phi\rangle^{2} & \geq \frac{4 k c}{\|\phi\|^{2}}\left(\langle a, h\rangle^{2}+\sigma\langle a, h\rangle\|\nabla a\|^{2}\right) \\
& \geq \frac{4 k c}{\|\phi\|^{2}}\left(\|h\|^{2}\left\|a_{\|}\right\|^{2}+\sigma\|h\|\|a\|\|\nabla a\|^{2}\right) \\
& \geq \frac{4 k c}{\|\phi\|^{2}}\|h\|^{2}\left\|a_{\|}\right\|^{2}+4 k c \sigma D_{3}\|a\|_{H^{1}}^{3}
\end{aligned}
$$

where $D_{3}>0$ is a constant.

Choose $\theta>0$ is such that $D_{1}-\theta D_{2} \equiv D_{4}>0$ and $\left\|a_{\perp}\right\|\left\|a_{\|}\right\| \leq \theta\left\|a_{\perp}\right\|^{2}+\frac{1}{\theta}\left\|a_{\|}\right\|^{2}$. Now, we can choose $k$ in such way that $\frac{4 k c}{\|\phi\|^{2}}\|h\|^{2}+\frac{\left\langle H_{c} h, h\right\rangle}{\|h\|^{2}}-\frac{D_{2}}{\theta}:=D_{5}>0$ to obtain

$$
\begin{aligned}
\left\langle H_{c} a, a\right\rangle+\frac{4 k c}{\|\phi\|^{2}}\langle a, \phi\rangle^{2} & \geq D_{5}\left\|a_{\|}\right\|^{2}+D_{4}\left\|a_{\perp}\right\|^{2}+4 k c \sigma D_{3}\|a\|_{H^{1}}^{3} \\
& \geq D^{\prime}\|a\|^{2}-D^{\prime \prime}\|a\|_{H^{1}}^{3}
\end{aligned}
$$


for some positive constants $D^{\prime}$ and $D^{\prime \prime}$.

Therefore, we have

$$
\left\langle H_{c} a, a\right\rangle+\frac{4 k c}{\|\phi\|^{2}}\langle a, \phi\rangle^{2} \geq \widetilde{D}_{1}\|a\|_{H^{1}}^{2}-\widetilde{D}_{2}\|a\|_{H^{1}}^{3},
$$

with $\widetilde{D}_{1}, \widetilde{D}_{2}>0$.

Finally using (A.25) in A.13) we obtain

$$
\Delta \widetilde{B}_{t} \geq c_{1}\|a\|_{H^{1}}^{2}-\sum_{j=1}^{2} c_{j}\|a\|_{H^{1}}^{j+2}-\sum_{j=1}^{2 k} c_{k, j}\|a\|_{H^{1}}^{j+2},
$$

where the constants are positive depending only on $c$.

Lemma A.4. The inequality (3.10) holds for all $t \in\left[0, t^{*}\right)$.

Proof. To show that the inequality (3.10) holds true for all $t \in\left[0, t^{*}\right)$, we follow the method introduced in Bona [12] and later used in Angulo et al [4, 5]. Consider the set $\mathcal{M}$ defined by

$$
\mathcal{M}=\left\{t: \text { the infimum in } \mathrm{A} .15 \text { is attained at finite values of } \gamma=\left(\gamma_{1}, \gamma_{2}\right)\right\} \text {. }
$$

It is evident from the discussion above that $[0, \tilde{T}] \subset \mathcal{M}$. Let $T_{1}$ be the largest value such that $\left[0, T_{1}\right) \subset \mathcal{M}$ and suppose that $T_{1}<t^{*}$. Now using (3.10) we get,

$$
\inf \Pi_{t}=\rho_{c}\left(\psi(\cdot, \cdot, t), \phi_{c}(\cdot, \cdot)\right)^{2} \leq \epsilon^{2} \leq \frac{1}{2}\left\|(\Delta+c)^{\frac{1}{2}} \phi_{c}\right\|^{2} .
$$

Since inf $\Pi_{t}$ is continuous on $t$ for all $t \in\left[0, t^{*}\right)$, we can find a $T>0$ such that

$$
\inf \Pi_{t}<\left\|(\Delta+c)^{\frac{1}{2}} \phi_{c}\right\|^{2}
$$

for all $t \in\left[T_{1}, T_{1}+T\right]$. But then Lemma A.3 implies that the infimum in A.15 is taken at finite values of $\gamma(t)$ and this contradicts the choice of $T_{1}$. Hence, we must have $T_{1}=t^{*}$ and this completes the proof of the theorem when $\mu(0)=1$.

\section{REFERENCES}

[1] M. J. Ablowitz and P. A. Clarkson, Solitons, Nonlinear Evolution Equations and Inverse Scattering, Cambridge University Press, Cambridge, 1991.

[2] J. P. Albert, Positivity properties and stability of solitary-wave solutions of model equations for long waves, Comm. Partial Differential Equations, 17 (1992), no. 1-2, 1-22. 
[3] J. P. Albert and J. L. Bona, Total positivity and the stability of internal waves in stratified fluids of finite depth, The Brooke Benjamin special issue (University Park, PA, 1989); IMA J. Appl. Math. 46 (1991), no. 1-2, 1-19.

[4] J. Angulo, J. L. Bona, F. Linares and M. Scialom, On the structure of singularities in solutions of the nonlinear Schrödinger equation for the critical case, $p=4 / n$, Nonlinear theory of generalized functions (1999), (M. Oberguggenberger et al., eds.) Chapman and Hall/CRC, Boca Raton, FL, $3-22$.

[5] J. Angulo, J. L. Bona, F. Linares and M. Scialom, Scaling, stability and singularities for nonlinear dispersive wave equations: the critical case, Nonlinearity 15 (2002), 759-786.

[6] J. Angulo and F. Linares, Global existence of solutions of a nonlinear dispersive model, J. Math. Anal. Appl. 195 (1995), 797-808.

[7] J. Angulo and M. Scialom, Improved blow-up of solutions of the generalized Boussinesq equation, Comp. Appl. Math. 18 (1999), 333-341.

[8] D. J. Benny, Long nonlinear waves in fluid flows, J. Math. Phys. (Stud. Appl. Math.) 45 (1966) $52-63$.

[9] Y.A. Berezin and V. I. Karpman, Nonlinear evolution of disturbances in plasmas and other dispersive media, Sov. Phys. JETP 24 (1967) 1049-1056.

[10] H. Biagioni and F. Linares, Well Posedness results for the modified Zakharov-Kuznetsov equation, Nonlinear equations: methods, models and applications (Bergamo, 2001), 181-189, Progr. Nonlinear Differential Equations Appl., 54, Birkhäuser, Basel, 2003.

[11] R. Blaha, E.W. Laedke and K.H. Spatschek, Collapsing states of generalized Korteweg-de Vries equations, Physica D, 40 (1989) 249-264.

[12] J. L. Bona, On the stability theory of solitary waves, Proc. Royal Soc. London, Ser A 344 (1975) $363-374$

[13] A. de Bouard, Stability and instability of some nonlinear dispersive solitary waves in higher dimension, Proc. Royal Soc. Edinburgh, Ser A 126 (1996) 89-112.

[14] D. B. Dix and W. R. McKinney, Numerical computations of self-similar blow-up solutions of the generalized Korteweg-de Vries equation, Differential and Integral Equations, 11 (1998) 679-723.

[15] A. V. Faminskii, The Cauchy problem for the Zakharov-Kuznetsov equation, Differential Equations 31 (6) (1995), 1002-1012.

[16] A. Hasegawa and C. Uberoi, The Alfvén wave, DOE Critical Rev. Ser. (Technical Information Center, U.S. Department of Energy, Springfield, VA, 1982.

[17] B. B. Kadomtsev and V. I. Petviashvili, On the stability of solitary waves in weakly dispersive media, Sov. Phys. Dokl., 15 (1970) 539-549.

[18] T. Kakutani and H. Ono, Weak nonlinear hydromagnetic waves in a cold collision-free plasma, J. Phys. Soc. Japan, 26 (1965) 1305-1318. 
[19] C. E. Kenig, G. Ponce and L. Vega, Well-posedness and scattering results for the generalized Korteweg-de Vries equation via the contraction principle, Comm. Pure Appl. Math., 46 (1993) 527620.

[20] E. A. Kuznetsov, A. M. Rubenchik, and V. E. Zakharov, Soliton stability in plasmas and hydrodynamics, Phys. Rep. 142 (1986), no. 3, 103-165.

[21] M. K. Kwong, Uniqueness of positive solutions of $\Delta u-u+u^{p}=0$ in $\mathbb{R}^{n}$, Arch. Rat. Mech. Anal. 105 (1989), 243-266.

[22] E. W. Laedke, L. Blaha, K. H. Spatschek and E. A. Kuznetsov, On stability of collapse in the critical case, J. Math. Phys. 33 (1992), 967-973.

[23] B. Li, Y. Chen and H. Zhang, Exact travelling wave solutions for a generalized Zakharov-Kuznetsov equation, Appl. Math. Comput. 146 (2003), no. 2-3, 653-666.

[24] F. Linares and A. Pastor, Well-posedness for the 2D modified Zakharov-Kuznetsov equation, Preprint 2008.

[25] S. Melkonian, S. A. Maslowe, Two-dimensional amplitude evolution equations for nonlinear dispersive waves on thin films Physica D 34 (1989), no. 1-2, 255-269.

[26] F. Merle, Existence of blow-up solutions in the energy space for the critical generalized KdV equation, J. Amer. Math. Soc. 14 (2001), 555-578.

[27] S. Monro, E.J. Parkes, The derivation of a modified Zakharov-Kuznetsov equation and the stability of its solutions, J. Plasma Phys, 62 (1999) (3) 305-17.

[28] S. Monro, E.J. Parkes, Stability of solitary-wave solutions to a modified Zakharov-Kuznetsov equation, J. Plasma Phys, 64 (2000) (3) 411 - 26.

[29] D. E. Pelinovsky and R. H. J. Grimshaw, An asymptotic approach to solitary wave instability and critical collapse in long-wave KdV-type evolution equations, Physica D, 98 (1996) 139-155.

[30] J. J. Rasmussen, and K. Rypdal, Blow-up in nonlinear Schrödinger equations. I. A general review, Phys. Scripta 33 (1986), no. 6, 481-497.

[31] K. Rypdal, and J. Rasmussen, Blow-up in nonlinear Schrödinger equations. II. Similarity structure of the blow-up singularity, Phys. Scripta 33 (1986), no. 6, 498-504.

[32] J.-C. Saut, Sur quelques dénéralisations de l'équation de Korteweg-de Vries, J. Math. Pures Appl. 58 (1979), 21-61.

[33] J.-C. Saut and N. Tzvetkov, The Cauchy problem for higher order KP equations, J. Diff. Equation 153 (1999), 196-222.

[34] H. Schamel, A modified Korteweg-de Vries equation for ion acoustic waves due to resonant electrons, J. Plasma Phys, 9 (1973) (3) 377-87.

[35] B. K. Shivamoggi, The Painlevé analysis of the Zakharov-Kuznetsov equation, Phys Scripta 42 No 6 (1990) 641-642.

[36] R. Sipcic and D. J. Benney, Lump Interactions and Collapse in the Modified Zakharov-Kuznetsov equation, Studies in Appl. Math. 105 (2000) 385-403. 
[37] M. Washimi and T. Taniuti, Propagation of ion-acoustic solitary waves of small amplitude, Phys. Rev. Lett. 17 (1966) 996-998.

[38] A. B. Wazwaz, Exact solutions with solitons and periodic structures for the Zakharov-Kuznetsov (ZK) equation and its modified form, Commun. Nonlinear Sci. Numer. Simul. 10 (2005), no. 6, 597-606.

[39] M. I. Weinstein, Nonlinear Schrödinger equation and sharp interpolation estimates, Comm. Math. Phys. 87 (1983), 567-576.

[40] M. I. Weinstein, On the structure and formation of singularities in solutions to nonlinear dispersive evolution equations, Comm. PDE 11 (1986), 545-565.

[41] M. I. Weinstein, Liapunov stability of ground states of nonlinear dispersive evolution equations, Comm. Pure Appl. Math. 39 (1986), 51-68.

[42] M. I. Weinstein, Solitary waves of nonlinear dispersive evolution equations with critical power nonlinearities, J. Diff. Eq. 69 (1987), 192-203.

[43] V. E. Zakharov and E. A. Kuznetsov, On three-dimensional solitons, Sov. Phys. JETP, 39 (1974), $285-286$.

Centro de Matemática, Universidade do Minho, 4710-057, Campus de Gualtar, Braga, Portugal.

E-mail address: mpanthee@math.uminho.pt

IMECC-UNICAMP, 13083-970, CAMPinas, SÃo PAUlo, Brazil.

E-mail address: scialom@ime.unicamp.br 